\title{
Dynamic Decision-Making Process of Evacuees during Post-Earthquake Evacuation near an Automatic Flap Barrier Gate System: A Broken Windows Perspective
}

\author{
Yu Song ${ }^{1}\left(\mathbb{D}\right.$, Jia Liu ${ }^{2}$ and Qian Liu ${ }^{1, * \mathbb{C}}$ \\ 1 School of Public Administration, Huazhong Agricultural University, Wuhan 430072, China; \\ songyu962@126.com \\ 2 School of Information and Safety Engineering, Zhongnan University of Economics and Law, \\ Wuhan 430073, China; liujia@zuel.edu.cn \\ * Correspondence: mathlq@163.com
}

Citation: Song, Y.; Liu, J.; Liu, Q. Dynamic Decision-Making Process of Evacuees during Post-Earthquake Evacuation near an Automatic Flap Barrier Gate System: A Broken Windows Perspective. Sustainability 2021, 13, 8771. https://doi.org/ $10.3390 /$ su13168771

\section{Academic Editors:}

Marta Borowska-Stefańska,

Michał Kowalski and

Szymon Wiśniewski

Received: 22 June 2021

Accepted: 31 July 2021

Published: 5 August 2021

Publisher's Note: MDPI stays neutral with regard to jurisdictional claims in published maps and institutional affiliations.

Copyright: (c) 2021 by the authors. Licensee MDPI, Basel, Switzerland. This article is an open access article distributed under the terms and conditions of the Creative Commons Attribution (CC BY) license (https:/ / creativecommons.org/licenses/by/ $4.0 /)$.

\begin{abstract}
The automatic flap barrier gate system (AFBGS) plays a critical role in building security, but it is more vulnerable to natural hazards than common exits (including power failure, due to earthquakes, and delayed evacuation, due to safety certification, etc.). This article considers a dynamic decision-making process of evacuees during post-earthquake evacuation near an AFBGS. An interesting metaphor, broken windows (BW), is utilized to interpret people's actual behavior during evacuation. A multi-stage decision-making mechanism of evacuees is developed to characterize the instantaneous transition among three defined stages: Habitual, mild, and radical states. Then, we build a modified three-layer social force model to reproduce the interaction between evacuees based on an actual post-earthquake evacuation. The simulations reveal that BW provides a contextualized understanding of emergency evacuation with a similar effect to the traditional metaphor. An earlier appearance of a mild rule breaker leads to a higher crowd evacuation efficiency. If evacuees maintain the state of broken windows behavior (BWB), the crowd evacuation efficiency can be improved significantly. Contrary to the criminological interpretation, the overall effect of mild BWB is positive, but the radical BWB is encouraged under the command of guiders.
\end{abstract}

Keywords: post-earthquake evacuation; decision-making process; automatic flap barrier gate system; broken windows; social force

\section{Introduction}

The transport infrastructure in congested regions often presents a vulnerability under natural disasters, such as an earthquake, fire, hurricane, etc. As a common, but serious, natural disaster, earthquakes principally result in shaking, ground rupture, structure collapse, etc., and destroy the stability of transport systems [1]. Many incidents with serious consequences occurred near extremely crowded areas, such as exits. Especially as an automatic multi-exit solution for metro stations, stadiums, universities, and commercial buildings, the automatic flap barrier gate system (AFBGS) is more vulnerable to hazards than common exits (e.g., a power outage caused by structural damage, time delay, due to access control, etc.). Post-incident analysis of the exit choice revealed that pedestrians attempted to evacuate through the same exit, ignoring less crowded points of egress during an emergency evacuation [2,3]. In addition to the characteristics of evacuees near common exits, a more complex and stepped decision-making process is witnessed near an AFBGS and has attracted considerable attention.

The decision-making process and evacuation behavior of evacuees are highly correlated to the group psychology and the structure of transport facilities under earthquakes [4,5]. Numerous previous studies are consistent with this observation. Scholars argue that pedestrians may not choose an efficient evacuation route after an earthquake [6]. 
The number, position, layout, and shape of exits have significant effects on exit choice and evacuation efficiency [7]. The placement of obstacles in the corner of exits can effectively reduce the evacuation time [8,9]. Environmental familiarity is also an important factor for pedestrian exit choice under uncertain information environments [3]. In addition, the limited visibility and hearing situation (i.e., smoke from fire, failure of lighting equipment, etc.) can change the individual exit choice [10,11]. While an AFBGS contains multiple adjacent exits with different structures, the exit choice of evacuees may change in phases influenced by combined factors. Several studies find that the crowd evacuation in a complex structure is not continuous, but presents phased characteristics. Bernardini et al. [12] state that a post-earthquake evacuation in a building can be divided into three stages (e.g., premovement, motion towards the target, and safe area reaching), which increases the probabilities of casualties. Staged evacuation is also widely used in optimization-oriented works, as different parts of the crowds may suffer different levels of risk [13,14]. Here, we try to distinguish the key factors for each phase of the individual decision-making process during post-earthquake evacuation near an AFBGS.

It is very difficult to explain the transformation of individuals' decision-making at different stages. Pedestrians make subjective assessments of their surroundings, based on several visible cues. Many conditions trigger a "individual unease" and are problematic for pedestrians, which may cause concerns about one's safety and evacuation efficiency [15]. Many of these cues in the crowd flow include overtaking, climbing over the railing, utilizing special channels, etc. An interesting multi-stage crowd evacuation was detected near an AFBGS in the video of a university library of Wuhan, China, in 2019 after an earthquake, as shown in Figure 1. At the initial period (stage 1), nearly all the evacuees only used the right exit (Figure 1a) according to the daily habit. With more pedestrians gathering near the door, an evacuee suddenly noticed another exit (Figure 1b, the red circle on the upper left) and ran to it. Then, this abnormal behavior was observed and imitated (Figure 1b,c) quickly (stage 2). More seriously, radical behaviors, climbing over the gates (Figure 1d), appeared later (stage 3). Due to the improper management or power failure caused by the earthquake, almost all the evacuees in the video did not use the access card, which strengthened the complex evolution of crowd dynamics [16]. The sudden appearance of an abnormal and dominant evacuation strategy (i.e., using another exit or climbing over the winged gate) breaks the routine, and stimulates the rapid imitation of which in the whole crowd.

Evacuees read these cues as a sign of "abnormal stimulations", which cause the erosion of the original cognition, and imitate others with obvious dominant evacuation strategy [15]. A metaphor is used to explicate this phenomenon. The broken windows theory (BWT) is a criminological or economic theory that visible signs of crime, illegal behavior, and social disorder create an environment that encourages further serious crimes [17]. However, narrow public reading may miss the very point of the approach and blames crimes for mere social disorders [16]. The highlight of BWT is the trigger or diffusion mechanism of some abnormal and noticeable behavior, and the corresponding metaphor is not confined to negative social phenomena (i.e., crime). In recent years, BWT has been extended to explain a series of positive social situations, such as prosocial behavior [18], economic creative destruction in a natural disaster [19], and environmentally responsible behaviors [20]. From a psychological point of view, the stimulation and diffusion of abnormal behaviors in the crowd (shown in Figure 1) are similar to the interpretation of the broken windows (BW) effect. Thus, BWT is used to provide a contextualized understanding of emergency evacuation to deduce the individual decisions under panic circumstances, and provide some novel insights to improve the evacuation efficiency by "encouraging certain abnormal behaviors". 


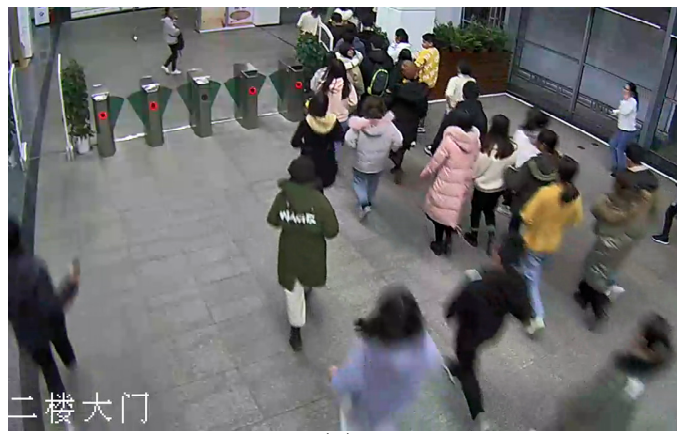

(a)

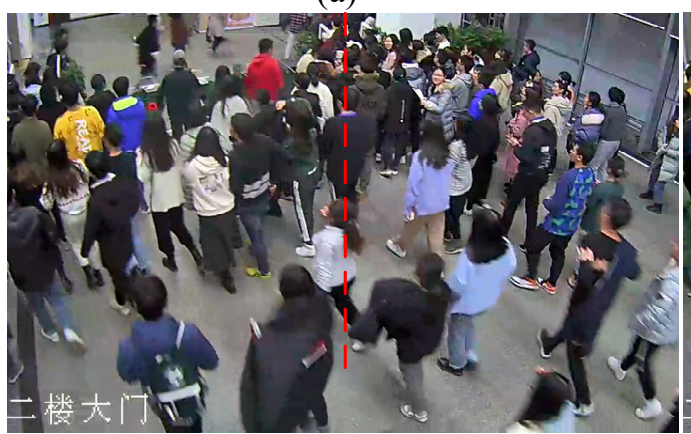

(c)

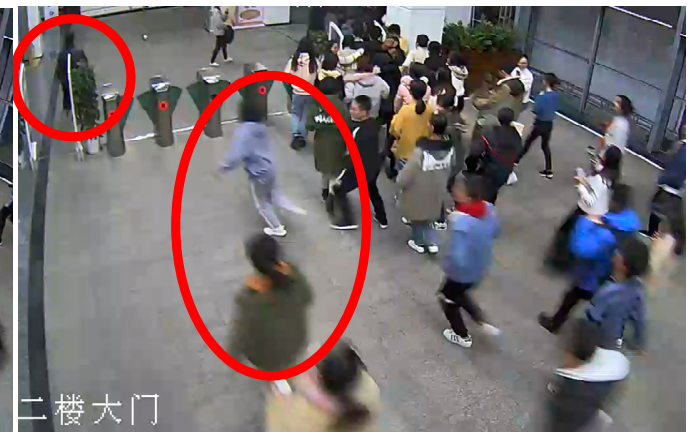

(b)

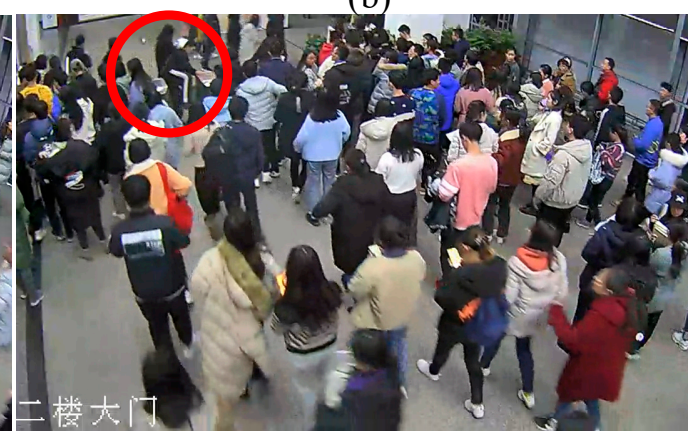

(d)

Figure 1. BW effect was recorded by the video of a university library in the moderate earthquake of Wuhan, China, December 2019. (a) all evacuees followed the daily habit and chosen the right door as the exit at stage $1 ;(\mathbf{b})$ an evacuee (the red circle at the top left corner) suddenly passed the left door, which was noticed by other evacuees (the red circles at the center); (c) more and more evacuees noticed the left door and chosen it as the exit, the daily habit was broken mildly at stage 2; (d) some impatient evacuees (the red circles) suddenly climbed over the closed winged gates, and this radical behavior was imitated by some other evacuees nearby at stage 3 .

The dynamic decision-making process and psychological state of evacuees are quite difficult to be incorporated in a physical pedestrian movement model. Some scholars have still made some progress in mathematical frameworks for pedestrian evacuation in a variety of cases (e.g., fire accident [21,22], earthquake, terrorist attack [23,24], flood disaster [25], etc.). The related works can be generally divided into two categories: The macroscopic continuum model and the microscopic discrete model. The former [26,27] characterizes the crowd motion as the homogeneous continuum flow, deducing a series of rigorous mathematical theories and numerical algorithms. The microscopic model can be further divided as: Social force model (SFM) [28,29], cellular automata model [30,31], lattice gas model [32,33], etc. Especially, SFM flexibly considers the dynamic interactions between individuals, deriving more reliable and realistic results compared to the macroscopic model [34]. Li et al. [35] modify the panic SFM and find that an evacuation leader is beneficial to maintain the calm and order of the crowd. Especially, a two-layer model is proposed to reproduce a real-life grouping situation in an earthquake evacuation [36].

In this paper, a BW perspective is introduced to help us to understand the evolutionary process of mild and radical evacuation behaviors. A three-layer SFM is developed to study the dynamic decision-making process of evacuees during post-earthquake evacuation near an AFBGS. The paper is organized as follows. Section 2 introduces the framework of the three-layer model, which includes the trigger mechanism of BW effect, individual decision, and crowd dynamics. In Section 3, we show that the new model can reproduce the realistic BW phenomenon recorded by the video. The validation and sensitivity analysis are conducted to explore the impact of the parameters. In Section 4, the utilities of evacuation strategies are compared to derive the policy suggestions. Section 5 concludes the influence mechanism of BW under post-earthquake evacuations. 


\section{Materials and Methods}

In accordance with the existing metaphor [17-20], the BW effect is defined as visible signs of abnormal/noticeable behavior in the crowd, which forms dominant strategies and encourages more followers. Metaphorically speaking, the first broken windows behavior (BWB) [37] refers to the trigger that changes the original state of the crowd. Involved in stress and chaos [38], the effect of BWB on individuals will be strengthened continuously. As shown in Figure 1, mild BWB (using the left door) and radical BWB (climbing over the gates) appear in the different stages of post-earthquake evacuation, and a three-layer SFM based on the staged BW effect is developed.

\subsection{Calculation Paradigm}

In terms of the surveillance video, the evacuation scenario is near an AFBGS. The schematic illustration of the scenario is shown in Figure 2. The typical evacuation strategies at such three stages are mainly divided into: Habitual evacuation, mild BWB, and radical BWB. Each evacuee $i$ considers three candidate strategies of exit choice: Passing the right door, passing the left door, and climbing over a closed barrier gate $m$. The choice of the right door represents the habitual evacuation strategy, and evacuees select the usual path. While the choice of the left door and that of closed barrier gate refer to the mild BWB and radical $\mathrm{BWB}$, respectively. The latter two are triggered and influenced by the rule breakers (RB). Based on the perception of the dynamic environment, each evacuee $i$ compares the disutility $U_{\text {right door }}^{i}, U_{\text {left door }}^{i}, U_{g_{m}}^{i}\left(g_{m}\right.$ denotes gate $\left.m\right)$ and chooses the strategy with the minimum disutility. The disutility $U_{k^{\prime}}^{i}, k \in\left\{\right.$ right door, left door, $\left.g_{m}\right\}$ is set as follows.

$$
U_{k}^{i}=\frac{T_{i \rightarrow k}}{\mu_{k}^{i}+\varepsilon}
$$

where $\mu_{k}^{i}$ is the perceived preference coefficient of strategy $k ; T_{i \rightarrow k}$ denotes the estimated travel time for individual $i$ to complete the strategy $k ; \varepsilon>0$ is the adjustment coefficient to prevent the denominator $\mu_{k}^{i}+\varepsilon$ from going to zero during the simulation. The computational details of $\mu_{k}^{i}$ and $T_{i \rightarrow k}$ are presented in Section 2.2.

The mild and radical RBs (MRB, RRB) are set to trigger different states: The first MRB breaks the habit mildly and selects the left door as the exit; the RRB climbs over a gate and escapes from the room. The whole evacuation process is divided into three stages:

- Stage 1. Evacuee $i$ with habitual state $\left(E^{1}\right)$ follows the daily habit and chooses the right door as the exit.

- $\quad$ Stage 2. Under the influence of MRB, evacuee $i$ decides whether he breaks the habit (comparing $U_{\text {right door }}^{i}$ and $U_{\text {left door }}^{i}$ ), and shifts to the mild BW state $\left(E^{2}\right)$.

- $\quad$ Stage 3. With the stimulation of RRB, evacuee $i$ decides whether he breaks the moral norm (upgrade to the radical BW state $E^{3}$ ) and imitates the RRB's behavior (comparing $U_{\text {right door }}^{i}, U_{\text {left door }}^{i}$ and $U_{g_{m}}^{i}$ ).

The calculation paradigm of the evacuation model is shown in Figure 2. 


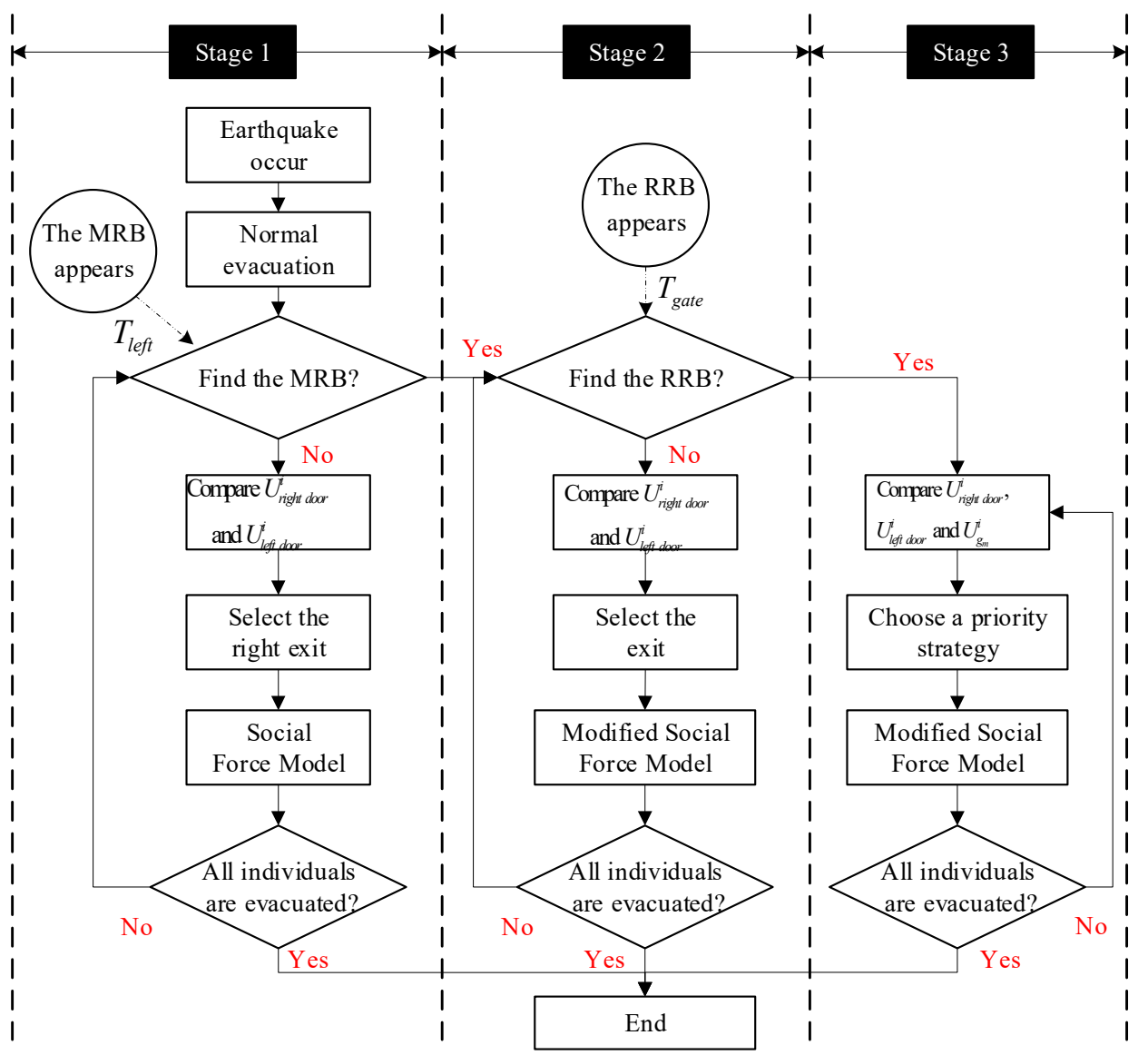

Figure 2. Calculation paradigm of the three-layer SFM.

\subsection{Multi-Stage BW Effect Model}

A new three-layer SFM is developed to simulate the crowd motion of pedestrians after earthquakes. The classical SFM [29] was introduced that an individual kept reasonable distances to other individuals and obstacles during the evacuation. For a pedestrian $i$ of mass $m_{i}(70 \mathrm{~kg})$, the actual velocity $\mathbf{v}_{i}$ at time $t$ is governed by the following equation:

$$
m_{i} \frac{d \mathbf{v}_{i}}{d t}=m_{i} \frac{v_{i}^{0} \mathbf{e}_{i}-\mathbf{v}_{i}}{\tau}+\sum_{j \in J_{i}} \mathbf{f}_{i j}+\sum_{w \in W} \mathbf{f}_{i w}
$$

where $\mathbf{f}_{i j}$ and $\mathbf{f}_{i w}$, respectively, denote the action forces that evacuee $j$ and wall $w$ exert on evacuee $i$. So that $i$ tries to keep a reasonable distance from evacuee $j$ and wall $w . J_{i}$ is the index set of the evacuees remaining in the room except evacuee $i, W$ is the index set of the walls inside the room. The coefficient $v_{i}^{0}$ is the desired speed, the unit vector $\mathbf{e}_{i}(t)$ pointing from $i$ to the target exit is the desired moving direction, $\tau>0$ is the characteristic time coefficient. $m_{i} \frac{v_{i}^{0} \mathbf{e}_{i}-\mathbf{v}_{i}}{\tau}$ is self-driven force meaning that evacuee $i$ tends to move with the desired speed in the desired direction by adjusting his actual velocity $\mathbf{v}_{i}$ within the characteristic time $\tau$. The action force $\mathbf{f}_{i j}$ that evacuee $j$ exerts on evacuee $i$ is calculated as follows.

$$
\mathbf{f}_{i j}=A_{i j} \exp \left(\frac{r_{i j}-d_{i j}}{B_{i}}\right) \mathbf{n}_{i j}+k g\left(r_{i j}-d_{i j}\right) \mathbf{n}_{i j}+\kappa g\left(r_{i j}-d_{i j}\right) \Delta v_{j i}^{t} \mathbf{t}_{i j}
$$

where $\mathbf{n}_{i j}$ is the unit vector pointing from the position of evacuee $j$ to that of evacuee i. $A_{i j} \exp \left(\frac{r_{i j}-d_{i j}}{B_{i}}\right) \mathbf{n}_{i j}$ represents the psychological tendency between $i$ and $j$ to stay away from each other. $r_{i j}$ is the sum of their body radius. $d_{i j}$ is the distance between two evacuees' centers of mass. $k g\left(r_{i j}-d_{i j}\right) \mathbf{n}_{i j}$ and $k g\left(r_{i j}-d_{i j}\right) \Delta v_{j i}^{t} \mathbf{t}_{i j}$ are the body force and 
the sliding friction force, respectively. They will arise when $i$ and $j$ touch each other, i.e., $d_{i j}<r_{i j}$. The function $g(x)=\max \{x, 0\}, \mathbf{t}_{i j}=\left(-n_{i j}^{2}, n_{i j}^{1}\right)$ is the tangential direction and $\Delta v_{j i}^{t}=\left(\mathbf{v}_{i}-\mathbf{v}_{j}\right) \cdot \mathbf{t}_{i j}$ is the tangential velocity difference. Similarly, the action force $\mathbf{f}_{i w}$ in Equation (2) between evacuee $i$ and wall $w$ is given by

$$
\mathbf{f}_{i w}=A_{i} \exp \left(\frac{r_{i}-d_{i w}}{B_{i}}\right) \mathbf{n}_{i w}+k g\left(r_{i}-d_{i w}\right) \mathbf{n}_{i w}-\kappa g\left(r_{i}-d_{i w}\right)\left(\mathbf{v}_{i} \cdot \mathbf{t}_{i w}\right) \mathbf{t}_{i w}
$$

For more details of $\mathbf{f}_{i j}$ and $\mathbf{f}_{i w}$, we refer to [29]. Finally, the desired direction $\mathbf{e}_{i}(t)$ is calculated as

$$
\mathbf{e}_{i}(t)=\frac{\mathbf{p}_{\text {exit-target }}(t)-\mathbf{p}_{i}(t)}{\left\|\mathbf{p}_{\text {exit-target }}(t)-\mathbf{p}_{i}(t)\right\|}
$$

where $\mathbf{p}_{i}(t)$ and $\mathbf{p}_{\text {exit-target }}(t)$ are the positions of evacuee $i$ and the target exit chosen by $i$ at time $t$, respectively. Clearly, the desired direction depends on the exit choice strategy. The desired direction $\mathbf{e}_{i}$ is used to describe different exit choice strategies in the three stages. Each strategy represents a target exit (right door, left door, or one gate). Integrating the multi-stage exit choice pattern with the SFM is essential to reveal the mechanism and effect of BWB during post-earthquake evacuation.

\subsubsection{Stage 1: Habitual Evacuation}

Under the impact of daily habits, as shown in Figure 1, the left door is overlooked, and the right door is selected as the unique exit. Then the desired direction $\mathbf{e}_{i}$ is set to point to the position of the right door at this stage. The motion of individual $i$ is calculated according to Equation (2).

\subsubsection{Stage 2: Habitual or Mild BWB Evacuation}

Referring to previous literature [39], an agent (MRB) is set to break the rules and select the left door as the exit at $T_{\text {left }}$. Some evacuees in the crowd may capture this abnormal phenomenon and suddenly notice the availability of the left door. Then the right door, together with the left door become the candidate exits. The daily habit (only using the right door) is mildly broken. It should be noted that, by this time, the radical behavior (climbing over barrier gates) does not appear because nearly all of evacuees are still patient. It takes a while for evacuees to get impatient and carry out radical actions. Therefore, in stage 2, evacuee $i$ compares the preference $\mu_{k}^{i}$ to decide the target exit and related desired direction. That is:

$$
\mu_{k}^{i}(t)=D H_{k}^{i}(t) \times F S_{k}^{i}(t), \quad k \in\{\text { right door, left door }\}
$$

where $D H_{k}^{i}(t)$ is a $0-1$ binary variable, which represents the habitual constraint of exit $k$ for pedestrian $i$ and characterizes the instantaneous transition from the habitual state to the MRB's state; a continuous variable $F S_{k}^{i}(t)$ indicates the stimulation of favorable strategy (SFS) on individual's decision making. The details are listed as follows.

(1) Daily habit $D H_{k}^{i}(t)$

Traffic in some countries and regions, including China, keeps right [29]. Following the basic behavior standard, the right door is regarded as the conventional exit and $D H_{\text {right }}^{i}(t) \equiv 1$. The MRB, as a visible sign of QBW stimulation, contributes to the deviation of daily habits - especially for using the left exit during the evacuation. Agent $i$ is affected by the MRB at $t_{\text {left }}^{i}\left(t_{\text {left }}^{i} \geq T_{\text {left }}\right)$. Then, the daily habit coefficient of the left door $D H_{\text {left }}^{i}(t)$ can be defined as:

$$
D H_{\text {left }}^{i}(t)=\left\{\begin{array}{ll}
0, & t<t_{\text {left }}^{i} \\
1, & t \geq t_{\text {left }}^{i}
\end{array} \text { the left door is not included in } i^{\prime}\right. \text { s decision set }
$$

However, not all individuals are influenced by the MRB immediately. The transition from $E^{1}$ to $E^{2}$ must satisfy at least one of the following two conditions. 
Condition 1: The evacuee directly observes that the left door is available.

There are evacuees (MRB or individuals with mild BWBs) near the left door in the semi-circular area $Q_{3}$ (see in Figure 3). Meanwhile, the vision of evacuee $i$ towards the left door is not covered by other pedestrians. That is:

$$
\frac{\pi\left(r_{\text {vision }}\right)^{2} N_{Q_{1}}}{S_{Q_{1}}} \leq \beta(0<\beta<1)
$$

where $Q_{1}$ is the edge area of vision towards the left door. $N_{Q_{1}}$ denotes numbers of agents in $Q_{1} . S_{Q_{1}}$ is the area of $Q_{1}$ calculated by visual angle $\theta_{\text {corner }}$ and the radius $r_{\text {vision }} . \beta$ is the shielding factor. When other agents' bodies shade the view, the individual cannot detect the situation near the left door (shown in Figure 3).

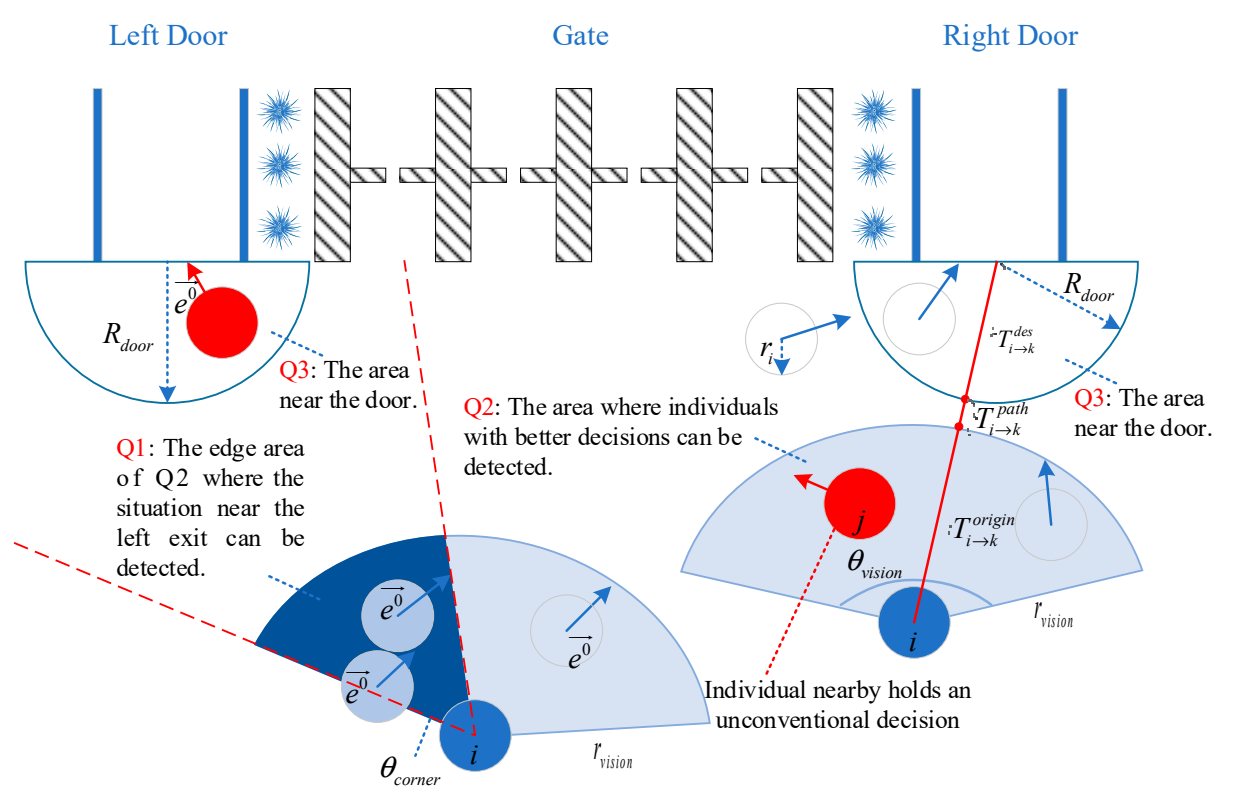

Figure 3. Schematic illustration of individuals with mild BWBs near the doors.

Condition 2: The evacuee realizes that the individual nearby holds an unconventional decision.

There is at least one individual $j$ with an unconventional decision in $Q_{2}$. Motion attributes of the agent $j$ is defined as follows.

$$
\left\{\begin{array}{l}
\mathbf{e}_{j}^{0} \rightarrow\left(x_{\text {left }}, y_{\text {left }}\right) \\
\arccos \left(\left\langle\mathbf{e}_{j}^{0}, \mathbf{v}_{j}\right\rangle /\left\|\mathbf{e}_{j}^{0}\right\|\left\|\mathbf{v}_{j}\right\|\right) \leq \omega \\
\left\|\mathbf{v}_{j}\right\| \geq \alpha
\end{array}\right.
$$

where $\mathbf{e}_{j}^{0} \rightarrow\left(x_{\text {left }}, y_{\text {left }}\right)$ denotes the direction of desired speed $\mathbf{e}_{j}^{0}$ points to the left door and $\mathbf{v}_{j}$ is the actual speed of agent $j$. The second condition in Equation (9) is that the angle between the actual moving and desired speed directions of agent $j$ is less than $\omega$. Meanwhile, the moving speed of agent $j$ is large enough (greater than the threshold $\alpha$ ). Then, $j^{\prime}$ s intention to choose the left door is observable for individual $i$.

(2) Stimulation of the partially-favorable strategy $F S_{k}^{i}(t)$ of door $k$

The herding effect $[31,40]$ is highlighted in the existing literature regarding seismic evacuation, which stresses that an agent often tries to keep the same moving direction and speed of the crowd. However, in a homogeneous environment, anomalies are always more likely to be observed and imitated [41,42]. As shown in Figure 1b, a small group was stimulated and quickly separated from the "sheep". They imitated the MRB while he ran 
away from the room quickly. The SFS is defined as a phenomenon that individuals are affected by a few predominant individuals. When the desired direction is limited within the crowd, individuals tend to be stimulated by a few abnormal evacuees nearby or who hold more favorable or efficient evacuation strategy.

The right door is always noticed by any evacuee during the evacuation and $F S_{\text {right }}^{i}(t) \equiv$ 1. For $t<t_{\text {left }}^{i}$, the evacuation advantage of the left door is not detected, so $F S_{\text {left }}^{i}(t)$ is set to 0 initially. At the time $t_{\text {left }}^{i}$, the availability of the left door is instantaneously perceived by individual $i$, resulting in a sudden and unexpected emotional stress. Prati et al. [43] stated that the emotional stress could reinforce the attraction of a strategy, if its utility increased suddenly from one time to another. Thus, to characterize the trigger effect, due to the sudden change of the perceived availability. $F S_{\text {left }}^{i}(t)$ is define as:

$$
F S_{\text {left }}^{i}(t)=1+\max \left\{\begin{array}{c}
\sum_{Q 2}^{N_{Q 2}\left(t_{\text {left }}^{i}\right)} \frac{\left\|\mathbf{v}_{q}\left(t_{\text {left }}^{i}\right)\right\|}{\delta_{1} \frac{d_{\text {iq }}\left(t_{\text {left }}^{i}\right)+\varepsilon}{\delta_{Q 2}\left(t_{\text {left }}^{i}\right)}}-\lambda_{1}\left(t-\mathbf{v}_{\text {left }}^{i}\left(t_{\text {left }}^{i}\right) \|\right. \\
\sum_{j=1}^{i} \frac{d_{i j}\left(t_{\text {left }}^{i}\right)+\varepsilon}{0}
\end{array}\right\}, t \geq t_{\text {left }}^{i}
$$

where $N_{\mathrm{Q} 2}\left(t_{\text {left }}^{i}\right)$ is the total number of pedestrians in $Q_{2}$ at time $t_{\text {left }}^{i}, N_{\mathrm{Q} 2}^{\text {left }}\left(t_{\text {left }}^{i}\right)$ is the number of pedestrian $q$ in $Q_{2}$ whose desired speed and moving speed point to the left door at time $t$, i.e., satisfying Equation (9). $d_{i q}\left(t_{\text {left }}^{i}\right)$ denotes the distance between $i$ and $q$. $\mathbf{v}_{q}\left(t_{\text {left }}^{i}\right)$ is the velocity of evacuee $q . \delta_{1}$ and $\varepsilon$ are the adjustment coefficients. Particularly, the fraction $\delta_{1} \sum_{q=1}^{N_{Q 2}^{\text {left }}(t)} \frac{\left\|\mathbf{v}_{q}(t)\right\|}{d_{i q}(t)+\varepsilon} / \sum_{j=1}^{N_{Q 2}(t)} \frac{\left\|\mathbf{v}_{j}(t)\right\|}{d_{i j}(t)+\varepsilon}$ means the herding effect of the crowd heading the left door $[43,44] . \delta_{1}$ presents individual difference of the psychological stimulation to SFS. This measures the abnormality degree of crowd behavior (left door strategy is abnormal and arresting for evacuee $i$ ) within the area of $Q_{2} . \lambda_{1}$ is the degradation coefficient of individual's SFS to the left door.

According to Equation (10), at $t=t_{\text {left }}^{i}$, the value of $F S_{\text {left }}^{i}(t)$ instantly jumps from zero to a real number greater than one. After $t_{\text {left }}^{i}$, the left door is included in the decision set. Individuals actively choose the optimal exit. However, since the right door and the left door have the same natural availability, the attraction of the left door declines gradually and reaches the same level as that of the right door eventually. The mathematical operation "max" of the second item on the right-hand side ensures $F S_{\text {left }}^{i}(t)$ is not less than 1 when $t \geq t_{\text {left }}^{i}$. Therefore, $F S_{\text {left }}^{i}(t)$ is larger than 1 at $t_{\text {left }}^{i}$, and then continues to decrease until equals to $1\left(F S_{\text {right }}^{i}(t)\right)$.

(3) Estimated evacuation time

The estimation evacuation time of target exit also affects individual's decision-making process. Accurately, the total evacuation time $T_{i \rightarrow k}$ is divided into three parts: Time to pass area $Q_{2}\left(T_{i \rightarrow k}^{\text {origin }}\right)$, time to pass area $Q_{3}\left(T_{i \rightarrow k}^{\text {des }}\right)$, and the rest $T_{i \rightarrow k}^{\text {path }}$ (shown in Figure 4). 
Left Door Gate Right Door

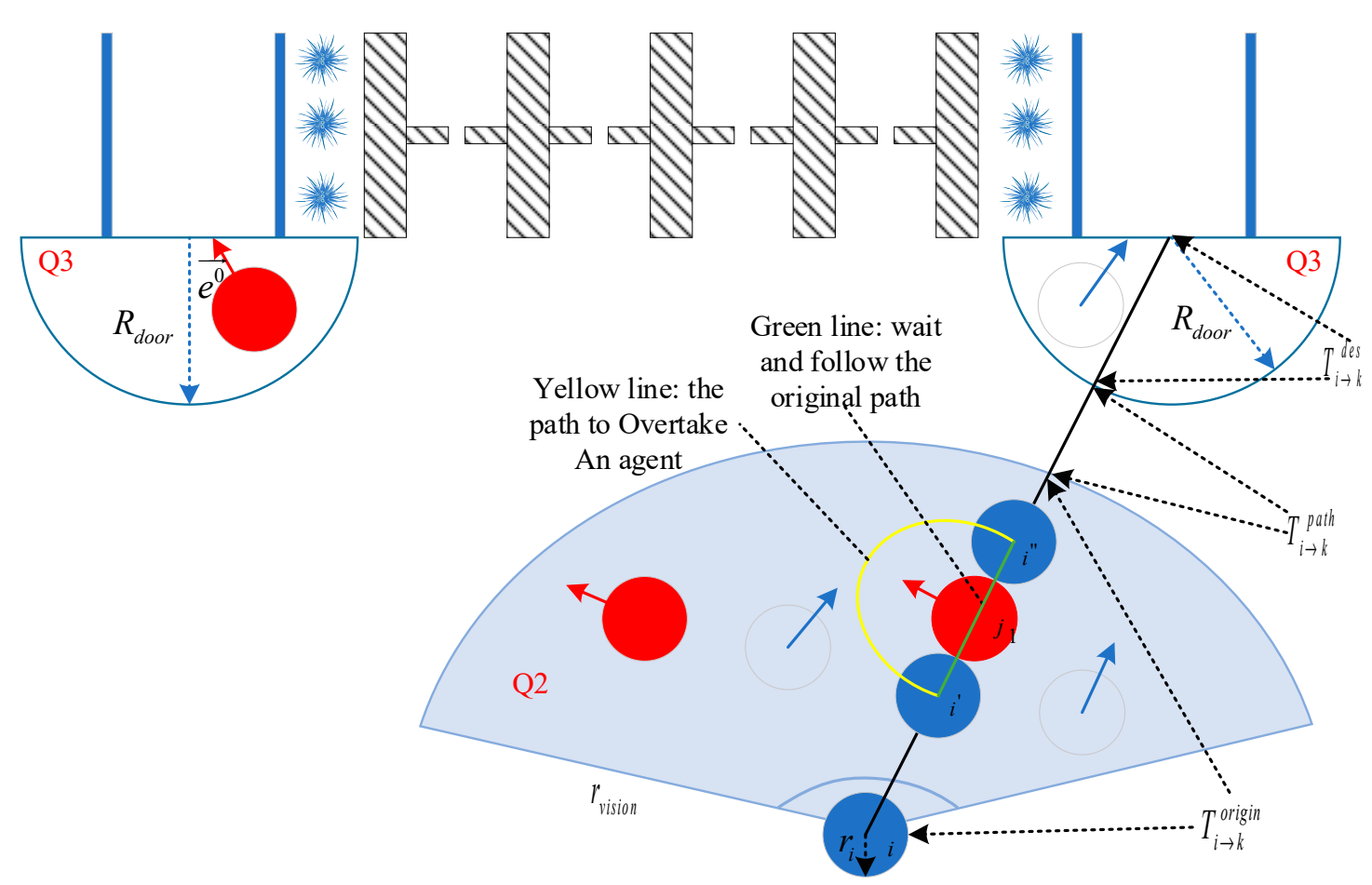

Figure 4. Schematic illustration of estimated evacuation time.

The estimated passing time of $Q_{2}$ for individuals is based on the velocity field and spatial distribution of evacuees moving to different exits. Assume that agent $i$ selects door $k$ ( $k \in\{$ right door, left door $\})$ as the target exit. Then, individuals with different decisions in their vision are divided into two categories. In addition to passing through the unobstructed area, agent $i$ may come into conflict with individuals $\left(A_{i \rightarrow k}^{\urcorner k}\right)$ who are moving to another exit, then evolves into overtaking or waiting behavior. The probabilities of overtaking and waiting are $\gamma$ and $1-\gamma$. Then the expected time to pass area $Q_{2}$ equals the sum of waiting, overtaking, and straight-line walking time.

$$
T_{i \rightarrow k}^{\text {origin }}=(1-\gamma) \times \frac{2 r_{i} N_{i \rightarrow k}^{\urcorner k}}{v_{i \rightarrow k}^{\urcorner k}+\vartheta}+\gamma \times \frac{\pi r_{i} N_{i \rightarrow k}^{\urcorner k}}{v_{i \rightarrow k}^{k}+\vartheta}+\max \left\{\frac{r_{\text {vision }}-\gamma \times 2 r_{i} N_{i \rightarrow k}^{\urcorner k}}{v_{i \rightarrow k}^{k}+\vartheta}, 0\right\}
$$

where $r_{i}$ is the radius of individual $i, v_{i \rightarrow k}^{k}$ is the average speed of agents moving to the same exit with $i$ in area $Q_{2}, N_{i \rightarrow k}^{\urcorner k}$ and $v_{i \rightarrow k}^{\urcorner k}$ denote the number and average speed of agents moving to the different exit from $i$ in $Q_{2}$. $\vartheta$ is the adjustment coefficient. $r_{v i s i o n}$ is the radius of $Q_{2}$. The three terms on the right-hand side of Equation (11) are the expected waiting, overtaking, and straight-line walking time, respectively. The numerical maximum value of the last term ensures that the item $\frac{r_{\text {vision }}-\gamma \times 2 r_{i} N_{i \rightarrow k} k}{v_{i \rightarrow k}^{k}+\vartheta}$ will not be less than 0 when too many agents $\left(N_{i \rightarrow k}^{\urcorner k}\right)$ choose the different exit compared to $i$.

The rest two regions tend to be a little far from $i$ and the evacuation time is roughly estimated. In area $Q_{3}$, pedestrians swarm towards the same exit, and the speed of the crowd tends to be homogeneous. $T_{i \rightarrow k}^{\text {des }}$ is defined as:

$$
T_{i \rightarrow k}^{\text {des }}=\frac{R_{\text {door }}}{v_{Q_{3}}}=R_{\text {door }} \times\left(\sum_{j \in P_{k}}\left\|\mathbf{v}_{j}\right\| / N_{i \rightarrow k}\right)^{-1}
$$


where $R_{\text {door }}$ is the radius of $Q_{3} . v_{Q_{3}}$ is the average speed of agents in the $Q_{3} . P_{k}$ is the set of agents in $Q_{3}$. $N_{i \rightarrow k}$ is the total number of agents in the $Q_{3} . T_{i \rightarrow k}^{p a t h}$ is defined as:

$$
T_{i \rightarrow k}^{p a t h}=\frac{d_{i \rightarrow k}-R_{d o o r}-r_{\text {vision }}}{\varphi v_{i}^{0}}
$$

where $d_{i-k}$ is the distance between agent $i$ and door $k . v_{i}^{0}$ is the desire speed. $\varphi$ is the conservative coefficient of the individual actual velocity. Considering the possibility of overlap of areas $Q_{2}, Q_{3}$ (see in Figure 4), the estimated time $T_{i \rightarrow k}$ is defined as:

$$
T_{i \rightarrow k}= \begin{cases}T_{i \rightarrow k}^{\text {origin }}+T_{i \rightarrow k}^{\text {path }}+T_{i \rightarrow k}^{\text {des }} & , d_{i \rightarrow k}>R_{\text {door }}+r_{\text {vision }} \\ T_{i \rightarrow k}^{\text {origin }}+\left(d_{i \rightarrow k}-r_{\text {vision }}\right) / v_{Q_{3}} & , R_{\text {door }}<d_{i \rightarrow k} \leq R_{\text {door }}+r_{\text {vision }} \\ d_{i \rightarrow k} / v_{Q_{3}} & , d_{i \rightarrow k} \leq R_{\text {door }}\end{cases}
$$

(4) Disutility and movement

Compare the disutility of two doors with Equation (15). The exit with the minimum disutility is chosen. The desired direction $\mathbf{e}_{i}$ in Equation (5) is set to this exit and the state of $i$ is updated to $E_{\text {right }}^{2}$ or $E_{\text {left }}^{2}$. Then the motion is calculated based on Equation (2).

$$
U_{k}=\frac{T_{i \rightarrow k}}{\mu_{k}^{i}+\varepsilon}=\frac{T_{i \rightarrow k}}{D H_{k}^{i}(t) \times F S_{k}^{i}(t)+\varepsilon}, k \in\{\text { left door, right door }\}
$$

\subsubsection{Stage 3: Habitual, Mildly BWB, or Radical BWB Evacuation}

At $T_{\text {gate }}\left(T_{\text {gate }}>T_{\text {left }}\right)$, a RRB near the AFBGS is set to climb over a gate. Then, evacuees satisfying both conditions below would be affected by the RRB: (1) $F S_{\text {left }}^{i}(t) \geq 1$. Transition to state $E^{2}$ is the basic condition for individuals to shift to stage 3. (2) Individuals near the RRB can transfer to the radical state and start to climb over a gate immediately $\left(\left\|\mathbf{P}_{i}-\mathbf{P}_{g_{m}}\right\| \leq R_{c l i m b}\right)$. The above settings are based on the video (see in Figure 1$)$. It should be noted that before entering Stage 3 , the evacuee must have entered stage 2 . When the RBB appears, the effect of radical BWB may be triggered, then passing the left/right door and climbing over winged gates are the candidate strategies for evacuees. Therefore, the target exit for agent $i$ in stage 3 is determined by comparing the exit disutility $U_{k}^{i}, k \in$ $\{$ left door, right door, gate $m\}$. The perceived preference $\mu_{k}^{i}$ for the left and right doors has been studied in Section 2.2.2 (Stage 2), and that for the winged gate in Stage 3 can be defined as:

$$
\mu_{\text {gate } m}^{i}(t)=M N_{g_{m}}^{i}(t) \times F S_{g_{m}}^{i}(t)
$$

where $g_{m}, m \in\{1,2,3,4\}$ denotes the different winged gates of the AFBGS. $M N_{g_{m}}^{i}(t)$ denotes the legal or moral constraint of individual $i$ about climbing over gate $m$. Similar to $F S_{\text {left door }}^{i}(t), F S_{g_{m}}^{i}(t)$ is set as a part of overall preference $\mu_{g_{m}}^{i}$ which changes continuously.

(1) Moral norm $M N_{g_{m}}^{i}(t)$

Each individual needs to swipe a card to enter the gate (as a one-way entrance) in daily life. Before the RRB appears, evacuees follow the regulations and laws $\left(M N_{g_{m}}^{i}(t)=0\right)$. After $t_{g_{m}}^{i}$, the time point that individual $i$ satisfies the trigger conditions, they tries to climb over the nearest gate to gain a competitive advantage. Their decision adds the option of climbing over the gate and $M N_{g_{m}}^{i}(t)=1$.

(2) Stimulation of the partially-favorable strategy $F S_{g_{m}}^{i}(t)$ of gate $g_{m}$

Walking on the left is only about habits in our hypothesis. $F S_{\text {left }}^{i}(t)$ is always no less than 1 after $t_{\text {left }}^{i}$. However, climbing over the gate is illegal or immoral behavior in daily life (e.g., a maximum fine of 1000 yuan for crossing the automatic ticket gate in Beijing Metro). In terms of the video, only a few students, which broke the regulations to choose the climbing strategy. Thus, $F S_{\text {gate }}^{i}(t)$ is set to stay low and decrease after $t_{g_{m}}^{i}$, even close to 0 . The radical BWB needs continuous "stimulation" to maintain a large positive value. 
Meanwhile, only when approaching the gate $\left(\left\|\mathbf{P}_{i}-\mathbf{P}_{g_{m}}\right\| \leq R_{c l i m b}\right)$, pedestrian $i$ may consider the strategy of climbing over a gate. For each empty gate $m, F S_{g_{m}}^{i}(t)$ is defined as:

$$
F S_{g_{m}}^{i}(t)=\max _{s \in\{1,2,3, \ldots, K\}}\left\{\delta_{3}+\delta_{2} \frac{N G_{s}}{N G}-\lambda_{2}\left(t-t_{s}^{i}\right), \quad \delta_{3}\right\} \times H\left(R_{c \lim b}-\left\|\mathbf{P}_{i}-\mathbf{P}_{g_{m}}\right\|\right)
$$

where NG denotes the number of gates. As RRB does not exist at every time step, the memory effect is considered that individual can remember the behavior of climbing over a gate (radical BWB) for the last $K$ times. At the last $s$-th time $(s \in\{1,2,3, \ldots, K\}), t_{s}^{i}$ is the moment when individual $i$ observes that other evacuees start to climb over the gate. $N G_{S}$ is the number of gates occupied by individuals at time $t_{s}^{i}$. In accordance with the Formula (8), $\delta_{2}$ and $\lambda_{2}$ are the psychological stimulation and the degradation coefficient of individual's SFS to the gates of AFBGS. $\delta_{3}$ denotes the basic value of $F S_{g_{m}}^{i}(t)$ which is less than 1 . It indicates that climbing over the gate is a sudden BWB, and the priority of which is lower than the evacuation strategy to use the left/right door, while the basic value of $F S_{k}^{i}(t)$ is 1 according to the Formula (8). Due to the moral constraint, for $t>t_{g_{m}}^{i}$, the psychological attraction $F S_{g_{m}}^{i}(t)$ tends to decrease gradually and become smaller than 1 eventually, i.e., $F S_{g_{m}}^{i}(t)<F S_{\text {left }}^{i}(t), F S_{\text {right }}^{i}(t) . \mathbf{P}_{i}, \mathbf{P}_{g_{m}}$ are the coordinates of the individual and gate $m$. The function $H(x)$ is 0 , if the pedestrians are far away from the gates $\left(R_{c l i m b}<\left\|\mathbf{P}_{i}-\mathbf{P}_{g_{m}}\right\|\right)$, and is otherwise equal to 1 .

(3) Estimated crossing time

Similarly, the estimation time for an evacuee to climb over a gate is defended as:

$$
T_{i \rightarrow g_{m}}=\frac{\left\|\mathbf{P}_{i}-\mathbf{P}_{g_{m}}\right\|}{\varphi v_{i}}+\mathrm{TC}
$$

where $\varphi(0<\varphi<1)$ is the conservative coefficient. $\left\|\mathbf{P}_{i}-\mathbf{P}_{g_{m}}\right\| / \varphi v_{i}$ denotes the walking time of individual $i$ to the exit. TC is the estimated time to climb over a gate.

(4) Disutility and movement

In stage 3 , the movement of individuals is determined by comparing the disutility of two exits $\left(U_{k}^{i}, k \in\{\right.$ left door, right door $\left.\}\right)$ and the four gates $\left(U_{g_{m}}^{i}, m=1,2,3,4\right)$, which are not occupied by other pedestrians currently. The disutilities of the left and right doors are presented in Section 2.2.2, and that of the gates is defined as

$$
U_{g_{m}}^{i}=\frac{T_{i \rightarrow g_{m}}}{\mu_{g_{m}}^{i}+\varepsilon}
$$

The exit with the minimum disutility is chosen by evacuee $i$, and the desired direction $\mathbf{e}_{i}$ in Equation (5) is set to this exit.

Based on the discrete evacuation model proposed in previous research $[9,45,46]$, the movement of the individual near a gate is simplified. The set $I(i \in I)$ represents individuals with the target $g_{m}$. Firstly, random numbers are generated for individuals, and the coordinate of the individual $i$ with the largest number is set to $\mathbf{P}_{g_{m}}\left(\mathbf{P}_{i}=\mathbf{P}_{g_{m}}\right)$ and the state is updated to $E^{3}$. Secondly, the individual $i$ is frozen for TC seconds to simulate the climbing behavior. Thirdly, remove the individual $i$ from the simulation environment and $g_{m}$ is remarked as empty.

\section{Results}

\subsection{Model Validation}

In this part, we focus on validating the three-layer SFM model developed in Section 2 and whether the model can reproduce the real post-earthquake evacuation process shown by Figure 1. The parameters of SFM are in accordance with Helbing's works [29,47,48]. Other parameters (refer to Appendix A) are properly set to match the realistic scenario. The shielding factor $\beta$ is set to 0.35 , and the perceptual threshold $\omega$ is set to $20^{\circ}$, which is 
according to the diopter of human eyes [49]. The threshold speed $\alpha=0.3 \mathrm{~m} / \mathrm{s}$, probability of overtaking $\gamma=0.9$, conservative coefficient $\varphi=0.9$ of actual velocity, estimated time $T C=0.5 \mathrm{~s}$ to climb over the gate, and the radius of climbing $R_{c l i m b}=1.5 \mathrm{~m}$ are estimated by the statistical information of realistic earthquake evacuation video $[12,35,50]$. Additionally, the coefficients of $P S_{\text {left door }}^{i}$, i.e., $\delta_{1}=0.3, \lambda_{1}=0.2$ and those of $P S_{g_{m}}^{i}$, i.e., $\delta_{2}=0.9, \delta_{3}=0.9$, $\lambda_{2}=0.2$, are estimated according to the simulation results (the sensitivity analysis will be conducted in Sections 3.1 and 3.2).

The configuration for the simulation scenario is as follows, using NetLogo as an agentbased programming language. As shown in Figure 5, a total of 150 pedestrians evacuates a room near an AFBGS. The AFBGS contains two doors and four gates (entrance using a swipe card), which is about $300 \mathrm{~m}^{2}$. The width of the two doors (pink and red), four gates (orange), and two pots of plant (green) are set to $1 \mathrm{~m}$ (see Figure 5). Then the time step is set to $1 \mathrm{~s}$. In each experiment, according to previous researches [51-53], 100 independent simulations were performed, and the average value was taken as the output result. Before the simulation began, 150 individuals were random placed away from AFBGS. An MRB and RRB were programmed exogenously into the simulation process.

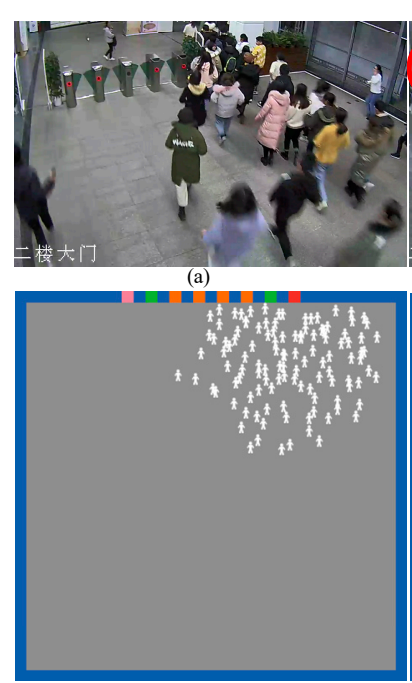

(e)
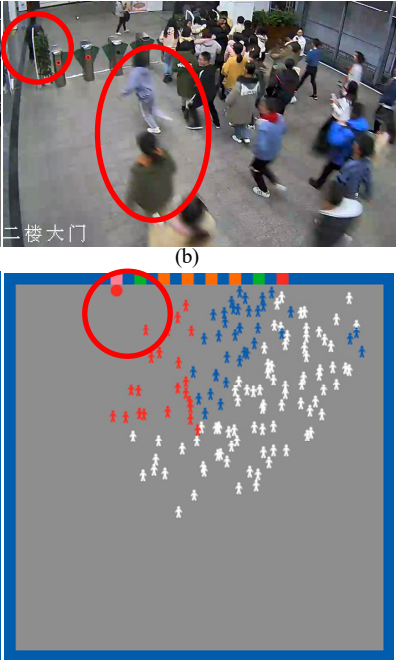

(f)
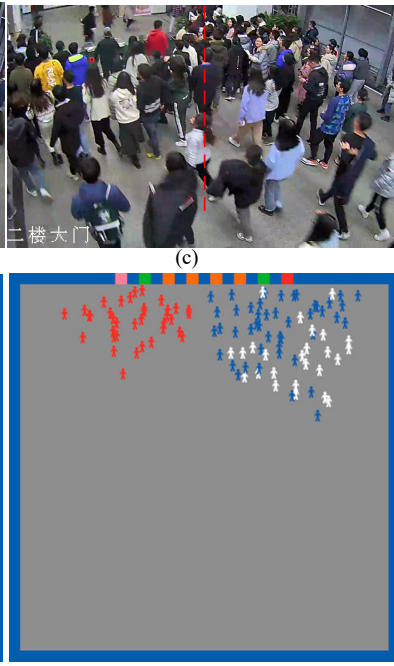

(g)
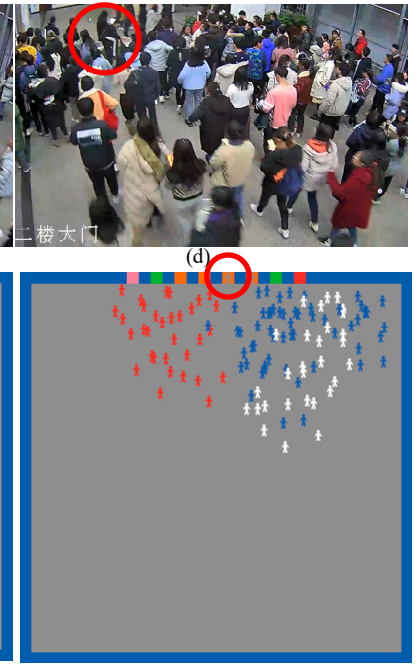

(h)

Figure 5. Screen shots of surveillance video and simulated evacuation scene. (a-d) show the typical screen shots of surveillance video corresponding to Figure 1. (e-h) show the evacuation simulation results corresponding to (a-d) respectively based on the three-layer SFM model.

We respectively record the evacuees with state $E^{1}$ (choose the right door), with state $E^{2}$ and choose the left door, with state $E^{2}$ and choose the right door, with state $E^{3}$ and climb over one-winged gate. The colors are set to white, red, blue, and grey, respectively, (as shown in Figure 5) for further comparison. Continuous spatial-temporal patterns are presented through the program. Comparing with the actual situation (see Figure 5a-d), the simulation scenarios of dynamic decision-making process of evacuees (see Figure $5 f-h$ ) show similar distributions. This means that the BW evacuation model developed in Section 2 can reproduce the real collective behavior in post-earthquake evacuation recorded by the video. The variation movement tactics and rules of individuals influenced by the MRB or RRB are analyzed in the following section.

\subsection{Numerical Simulation of the MRB's Behaviors}

(1) Effect of trigger time $T_{\text {left }}$

The trigger time $T_{l e f t}$ indicates the moment that the MRB appears, and it show great importance to the appearance of mild BWBs. As shown in Figure 6, the decrease of $T_{\text {left }}$ makes the total evacuation time (TET) shorter and improves the evacuation efficiency. The 
appearance of MRB at the beginning of evacuation shortens the TET (near $30 \mathrm{~s}$ ) compared with the control group (the actual evacuation). Meanwhile, the earlier detection of MRB increases the utilization of the left door for evacuees. The number of evacuees using the left door shows a linear growth with the decrease of $T_{l e f t}$, which is stable at about half of the total number of evacuees.

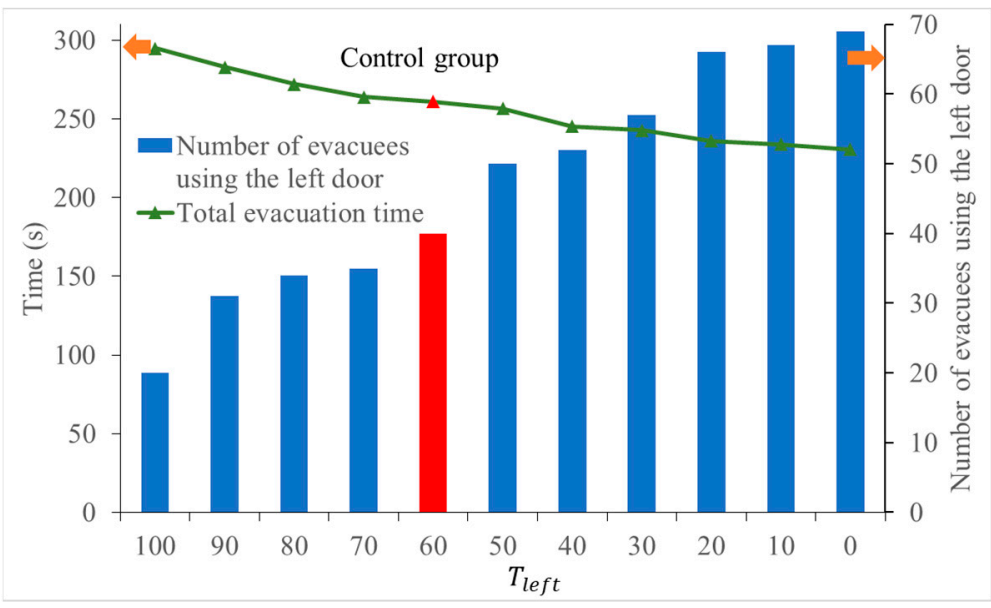

Figure 6. TET and the total number of evacuees using the left door for different trigger time $T_{\text {left }}$. The bar chart shows the number of evacuees using the left door, and the line graph indicates the influence of $T_{\text {left }}$ on TET.

(2) Effect of the stimulation of favorable strategy $F S_{k}^{i}(t)$

The SFS is particularly obvious in the homogeneous system, and affects the individual decision-making process. It is regarded as the stimulation of the MRB or individuals choosing the left door. To investigate the influence of $F S_{\text {left }}^{i}(t)$ after the appearance of the MRB, the usage of the left door is measured when changing the value of stimulation coefficient $\delta_{1}$ and degradation coefficient $\lambda_{1}$ (see in Figure 7).
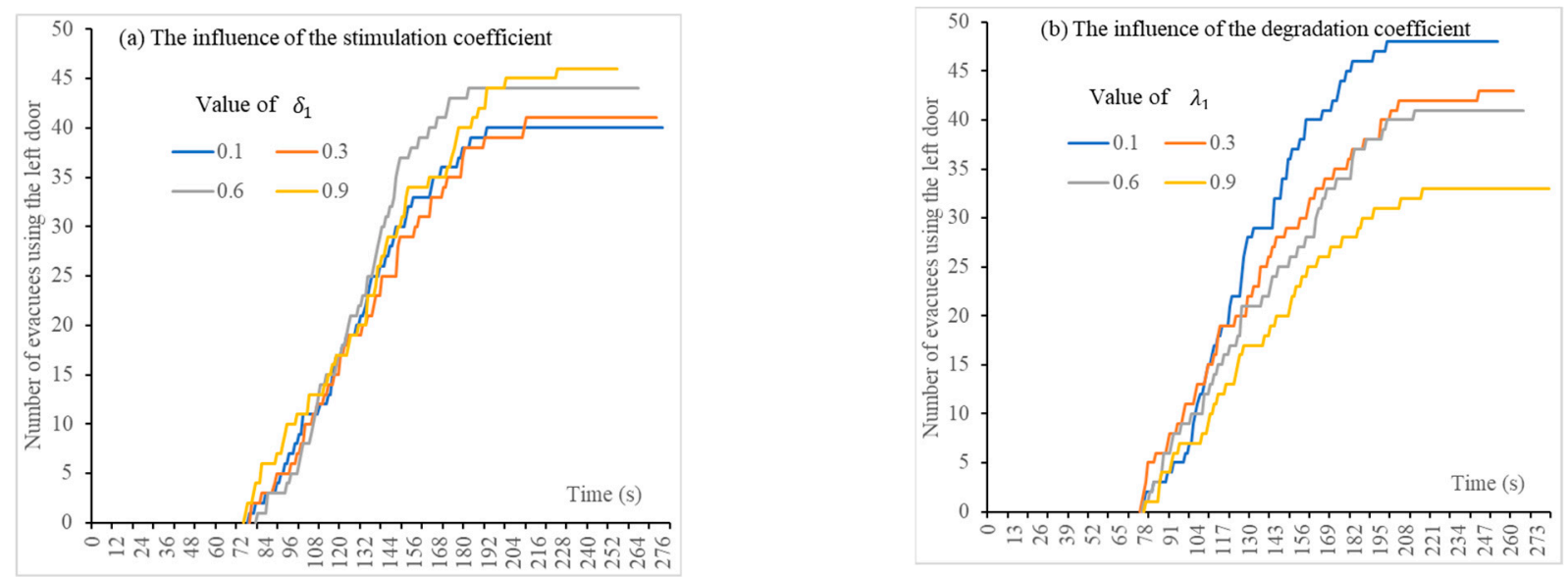

Figure 7. The total number of evacuees using left door for different values of (a) $\delta_{1}$ and (b) $\lambda_{1}$.

$\delta_{1}$ refers to the psychological stimulation of SFS (the initial value is 0.3 ). As shown in Figure $7 \mathrm{a}$, the evacuation scenarios are similar. With a larger $\delta_{1}$, more individuals transfer to state $E^{2}$ and utilize the left door. The TET becomes shorter, while the efficiency of the two exits (especially the left door) are improved. When $\delta_{1}$ is 0.9 , the growth rate of the usage of left door is the largest. More individuals in state $E^{1}$ are affected by the MRB, and then transfer to $E^{2}$ and choose the left door as the exit. The process of transition is greatly accelerated. 
$\lambda_{1}$ refers to the decline of an individual's BW psychology to MRB. The change of $\lambda_{1}$ has a reverse effect on the evacuation process. Figure $7 \mathrm{~b}$ shows that the numbers of evacuees using the left door is higher with a lower value of $\lambda_{1}$. The cumulative increase of evacuees choosing the left door strengthens the mutual psychological stimulation in the crowd. More evacuees ignore the daily habits and make the optimal decision by comparing the disutility. Therefore, the evacuation time is reduced.

\subsection{Numerical Simulation of the RRB's Stimulation}

(1) Effect of trigger time $T_{\text {gate }}$ and crossing time TC

Here in this section, we focus on the effect of the time RRB appears (trigger time $T_{\text {gate }}$ ) and the time evacuees climb over the gates of AFBGS (crossing time TC) on evacuees' radical BWB. The impact of trigger time $T_{\text {gate }}\left(T_{\text {gate }}>T_{\text {left }}\right)$ on the TET is generally unnoticeable (as shown in Figure 8). For $T_{\text {gate }} \geq 70 \mathrm{~s}$ (after the scenario one), as $T_{\text {gate }}$ increases, the total number of evacuees climbing over the gates in the whole evacuation (TEG) decreases significantly and TET slightly increases. In addition, TEG continues to increase, while $T_{\text {gate }}$ increases from $70 \mathrm{~s}$ to $90 \mathrm{~s}$, and reaches the maximum value at $T_{\text {gate }}=90 \mathrm{~s}$. After that, TEG presents a steady decline eventually. It can be seen from Figure $8 \mathrm{~b}$ that the impact of estimated crossing time (TC) on TET is relatively small. Moreover, as TC increases, the individual cost of climbing over a gate will also increase, and then fewer evacuees will choose gates as the exit.
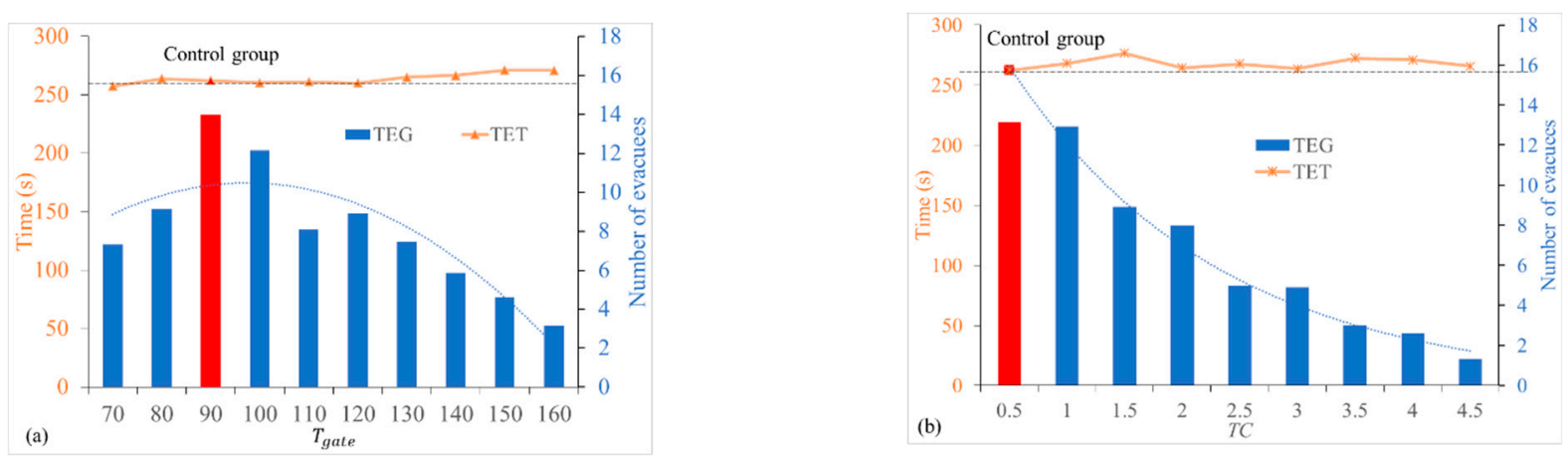

Figure 8. TET and TEG for different values of $T_{\text {gate }}$ and TC. (a) the line graph shows the influence of $T_{\text {gate }}$ on TET, and the bar chart shows the influence of $T_{\text {gate }}$ on TEG. (b) the line graph and bar chart show the infulence of TC on TET and TEG respectively.

(2) Effect of the stimulation of favorable strategy $F S_{g_{m}}^{i}(t)$

Here in this section, we focus on the effect of the psychological effect and the degradation coefficient of individual's SFS to the gates of AFBGS $\left(\delta_{2}, \delta_{3}\right.$, and $\left.\lambda_{2}\right)$ on the SFS, TET, and the evacuation strategies of evacuees. The simulation results show that the impact of $\delta_{2}$ is rather small, so we only report the results about $\delta_{3}$ to study the system performance after trigger time $T_{\text {gate }}$, as shown in Figure 9a. As $\delta_{3}$ increases, TET varies from $260 \mathrm{~s}$ to $270 \mathrm{~s}$, implying that the transition is moderate. Furthermore, the number of evacuees using the gates during simulation process (NEUG) changes significantly with different values of $\delta_{3}$. The changing curves of NEUG is unimodal and declines rapidly if $\delta_{3}$ is small $\left(\delta_{3}=0.1\right.$ or 0.3$)$, and it is multimodal and fluctuates repeatedly when $\delta_{3}$ is large $\left(\delta_{3}=0.6\right.$ or 0.9 ). A higher level of $\delta_{3}$ (for example, comparing $\delta_{3}=0.9$ and $\delta_{3}=0.1$ ) generally results in a larger NEUG, as well as a longer time for using gates during the evacuation process. This implies that $\delta_{3}$ has a noticeable effect on the exit strategies of evacuees. 

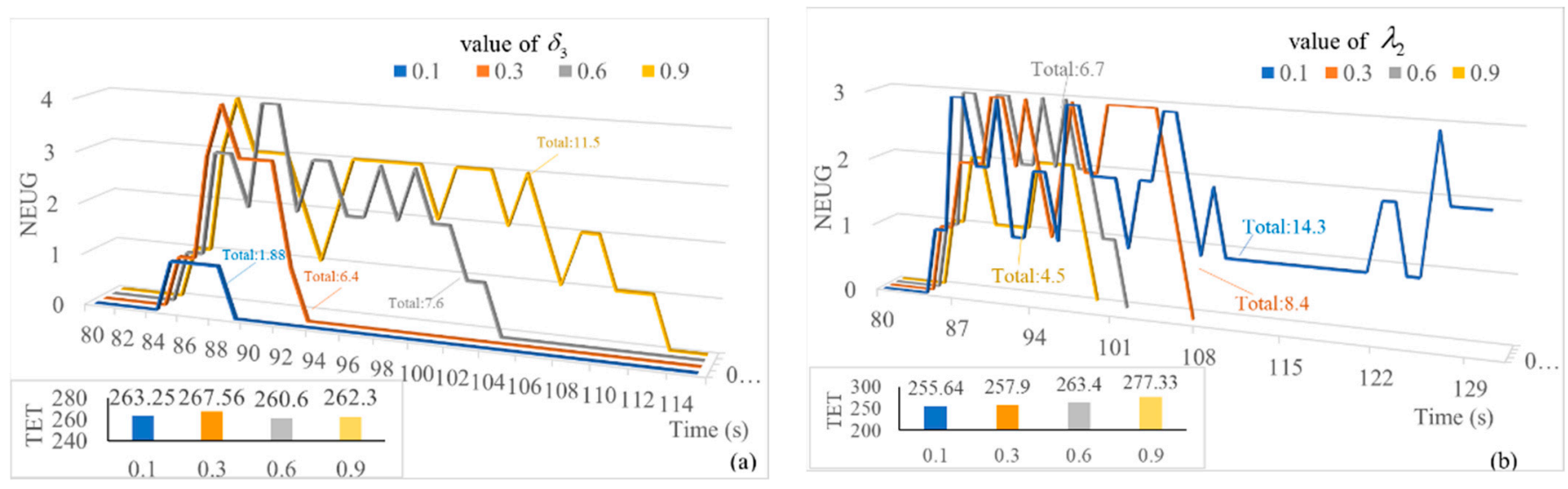

Figure 9. TET and NEUG for different values of $\delta_{3}$ and $\lambda_{2}$. (a) the line graph and bar chart show the influence of different values of $\delta_{3}$ on NEUG and TET. (b) the line graph and bar chart show the influence of different values of $\lambda_{2}$ on NEUG and TET.

The effect of degradation coefficient $\lambda_{2}$ is presented after trigger time $T_{\text {gate }}$ in Figure $9 \mathrm{~b}$. As $\lambda_{2}$ increases from 0.1 to 0.9 , TET increases from $255 \mathrm{~s}$ to $277 \mathrm{~s}$. Thus, the effect is relatively apparent and the decrease of $\lambda_{2}$ can improve the total evacuation efficiency. Meanwhile, as $\lambda_{2}$ increases, the number of evacuees using gates also increases and the growth rate is positively related with $\lambda_{2}$. In addition, a lower level of $\lambda_{2}$ (for example, comparing $\lambda_{2}=0.9$ and $\lambda_{2}=0.1$ ) generally results in a longer time for using gates during the whole evacuation process. The decrease of $\lambda_{2}$ can rise the utilization rate of gates.

\section{Discussion}

People will not take the initiative to violate the rules or laws to obtain convenience in daily life $[39,54]$. Even in the classical "broken windows" experiments, the cars offered was also slightly damaged (not new) — which then caused a deterioration [17,37]. Pedestrians are told to regulate their own behaviors under the influence of moral or legal norms, for example do not walk on the left and do not climb over the gate. It is a kind of habitual action, especially for the college students in the video, which hold the basic principle of "walking on the right" at the beginning of evacuation. External stimulation of the MRB or RRB is necessary for individuals to form a psychological hint of a better evacuation decision. Then, all possibilities of exit will be tested and identified.

Previous searchers put forward many psychological theories to describe evacuees' herb behavior as "the need to behave in the same way as everyone else does" [40,41], which is also found in economics and business fields. Similar phenomena occur during the evacuation, evacuees prefer the crowded door as the exit. However, the video indicates people follow the minorities with an advantage strategy than their original one. In addition to the empirical studies, this kind of BW psychology spreads quickly after the breaker appears.

The BWT is highly influential. The impact of some abnormal behaviors will lead to more followers [17-20], no matter it is positive or negative. In this study, although the BWB is an abnormal or even immoral behavior in daily life, it is encouraged as the evacuation efficiency improves to a certain extent. The mild BWBs significantly reduce the TET and the environmental risk is controllable, while the two doors can be regarded as one-way exits to eliminate the influence of daily habits under emergencies. In contrast, the radical BWBs do not significantly improve the overall evacuation efficiency, due to the time and ability of climbing behavior. Meanwhile, there are secondary risks when climbing over the gate for evacuees, e.g., tripped over the winged door, building collapsed, due to earthquake, etc. $[1,4]$

Contrary to the criminological metaphor, the BWB under emergencies is not just about catastrophic consequences. A mild or radical breaker may lead to different evacuation 
results. The positive BWBs refer to breaking away from convention under the constraints of rules, e.g., staying calm and proactive about optimizing the environment, moving obstacles, offering assistance, etc. The negative BWBs refer to the violations mentioned above. Considering the effect of $\mathrm{BWB}$, optimization strategies are proposed. The corresponding evacuation results are shown in Figure 10.

- Strategy 1: Conducting the mild BWB initially. An MRB appears initially, and the individuals imitate and follow the MRB.

- Strategy 2: Improving the ability to break the routine (mild BWB) under evacuation. Through safety education, evacuation exercises, slogans, etc., increase the ability of individuals to continuously and actively break the routine (mild BWB) to promote evacuation efficiency. But preventing them from exhibiting radical behaviors (climbing over the gates). The efficiency of Strategy 2 is demonstrated in Figure 7.

- Strategy 3: Conducting the radical BWB initially with a guider. A guider (see in Figure 10 ) is set near the gate to help individuals to swipe the card.

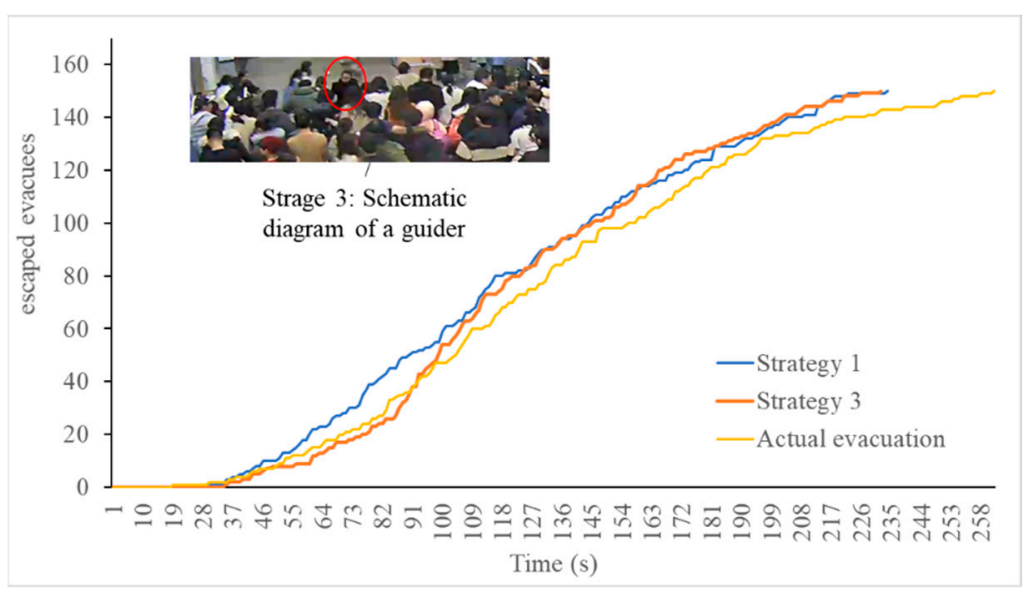

Figure 10. Number of evacuees escaped from the room with optimization strategies.

The results in Figure 10 tell an interesting story. The mild BWB only or the radical behavior under the command of certain guiders is recommended. Firstly, extreme behavior is not encouraged in real evacuation. However, effective guidance can reduce the negative impacts (e.g., risk of falls and stampede) of individual radical behaviors and increase evacuation efficiency. Secondly, if there is no guider, it is valuable for setting the slogans near the AFBGS and reinforcing the safety education to extend the time of mild BWB of evacuees. Low risk resources (e.g., new exits or paths) in the circumstance are encouraged to be rapidly integrated. The manager can stimulate the evacuees to cause the thought of breaking the routine slightly (generating MRBs) through the environmental stimulations (such as slogans and signs). Even in non-seismic zone, managers should be alert of natural hazards, and ensure the availability and connectivity of facilities.

\section{Conclusions}

In this work, we describe a dynamic decision-making process of evacuees during postearthquake evacuation near an AFBGS. The BWT is utilized to provide a contextualized understanding of the pedestrian evacuation behavior. A multi-stage individual exit choice mechanism is developed to characterize the instantaneous transition among different states (habitual state, mild BWB state, and radical BWB state). A three-layer social force model is proposed to reproduce the pedestrian evacuation process under different scenarios. Simulation results show that: (1) An earlier appearance of the first MRB leads to a higher crowd evacuation efficiency. While the impact of the trigger time of RRB is not significant. (2) If evacuees maintain the state of BWB, e.g., increasing the psychological perception of mild BWB, decreasing the psychological decay of mild and radical BWBs, the crowd 
evacuation efficiency can be improved significantly.(3) The mild BWB and radical BWB under the command of guiders are recommended. Whereas, effective guidance can reduce the negative impacts (e.g., risk of falls and stampede) and increase evacuation efficiency.

Author Contributions: Conceptualization, Q.L. and Y.S.; methodology, Q.L.; software, Y.S.; writingoriginal draft preparation, Y.S.; writing-review and editing, J.L.; project administration, Q.L. All authors have read and agreed to the published version of the manuscript.

Funding: This research is supported by the National Natural Science Foundation of China (No. 72071212), Natural Science Foundation of Hubei Province (No. 2020CFB518, 2020CFB321, 2020CFB315), the Fundamental Research Funds for the Central Universities (No. 2662019QD025, 2662018QD035).

Informed Consent Statement: Informed consent was obtained from all subjects involved in the study.

Data Availability Statement: The data presented in this study are available on request from the corresponding author. The videos are not publicly available as informed consent obtained from all subjects in the video specifically applies to this study. We need their permissions for further studies.

Conflicts of Interest: The authors declare no conflict of interest.

\section{Appendix A}

\begin{tabular}{|c|c|c|c|}
\hline Parameters & Reference & Notation & Initial Value \\
\hline \multicolumn{4}{|c|}{$\begin{array}{l}\text { Normal evacuation } \\
\end{array}$} \\
\hline Number of agents & & $N$ & The parameters of SFM are in accordance with [29] \\
\hline \multicolumn{4}{|c|}{$B W$ effect of $M R B$} \\
\hline Appearance time of the mild breaker & Video-based statistics & $T_{\text {left }}$ & 60 \\
\hline Radius of $Q_{3}$ & Video-based statistics & $R_{\text {door }}$ & $1.5 \mathrm{~m}$ \\
\hline Adjustment coefficient of $U_{k}^{i}$ & Mathematical principles & $\varepsilon$ & 0.1 \\
\hline Shielding factor & & $\beta$ & 0.35 \\
\hline Radius of core view & According to the human eye & $r_{\text {vision }}$ & $3 \mathrm{~m}$ \\
\hline Edge angle of view & diopter [49] & $\theta_{\text {corner }}$ & $120^{\circ}$ \\
\hline $\begin{array}{l}\text { Threshold of the angle between the actual and } \\
\text { desired speed }\end{array}$ & & $\omega$ & $20^{\circ}$ \\
\hline Threshold speed to be observed & Video-based statistics & $\alpha$ & $0.3 \mathrm{~m} / \mathrm{s}$ \\
\hline \multirow{2}{*}{ Stimulation coefficient of $F S_{\text {left door }}^{i}$} & Video-based statistics & $\delta_{1}$ & 0.3 \\
\hline & Video-based fitting & $\varepsilon$ & 0.1 \\
\hline Degradation coefficient of $F S_{\text {left door }}^{i}$ & Video-based fitting & $\lambda_{1}$ & 0.2 \\
\hline Probability of overtaking & Video-based statistics & $\gamma$ & 0.9 \\
\hline Conservative coefficient of actual velocity & Video-based statistics & $\varphi$ & 0.9 \\
\hline Adjustment coefficient of $T_{i \rightarrow k}^{\text {origin }}$ & Mathematical principles & $\vartheta$ & 0.1 \\
\hline \multicolumn{4}{|c|}{$B W$ effect of $R R B$} \\
\hline Appearance time of the radical breaker & Video-based statistics & $T_{\text {gate }}$ & 73 \\
\hline Estimated time to climb over the gate & Video-based statistics & TC & $0.5 \mathrm{~s}$ \\
\hline Radius of climbing & Video-based statistics & $R_{c l i m b}$ & $1.5 \mathrm{~m}$ \\
\hline \multirow{3}{*}{ Adjustment coefficient of $F S_{g_{m}}^{i}$} & Video-based fitting & $\delta_{2}$ & 0.9 \\
\hline & Video-based fitting & $\delta_{3}$ & 0.9 \\
\hline & Video-based fitting & $\lambda_{2}$ & 0.2 \\
\hline
\end{tabular}

\section{References}

1. Song, Y.; Xie, K.; Su, W. Mechanism and strategies of post-earthquake evacuation based on cellular automata model. Int. J. Disaster Risk Reduct. 2019, 34, 220-231. [CrossRef]

2. Sime, J.D. Affiliative behaviour during escape to building exits. J. Environ. Psychol. 1983, 3, 21-41. [CrossRef]

3. Kinateder, M.; Comunale, B.; Warren, W.H. Exit choice in an emergency evacuation scenario is influenced by exit familiarity and neighbor behavior. Saf. Sci. 2018, 106, 170-175. [CrossRef] 
4. Lu, X.; Yang, Z.; Cimellaro, G.P.; Xu, Z. Pedestrian evacuation simulation under the scenario with earthquake-induced falling debris. Saf. Sci. 2019, 114, 61-71. [CrossRef]

5. Xiao, M.L.; Zhang, Y.; Liu, B. Simulation of primary school-aged children's earthquake evacuation in rural town. Nat. Hazards 2017, 87, 1783-1806. [CrossRef]

6. Alexander, D. Behavior during earthquakes: A southern Italian example. Int. J. Mass Emerg. Disasters 1990, 8, 5-29.

7. Yue, H.; Guan, H.; Shao, C.; Zhang, X. Simulation of pedestrian evacuation with asymmetrical exits layout. Phys. A Stat. Mech. Its Appl. 2011, 390, 198-207. [CrossRef]

8. Haghani, M.; Sarvi, M. Simulating pedestrian flow through narrow exits. Phys. Lett. A 2019, 383, 110-120. [CrossRef]

9. Guo, R.Y.; Huang, H.J.; Wong, S.C. Route choice in pedestrian evacuation under conditions of good and zero visibility: Experimental and simulation results. Transp. Res. Part B Methodol. 2012, 46, 669-686. [CrossRef]

10. Cao, S.; Fu, L.; Wang, P.; Zeng, G.; Song, W. Experimental and modeling study on evacuation under good and limited visibility in a supermarket. Fire Saf. J. 2018, 102, 27-36. [CrossRef]

11. Xue, S.; Shi, X.; Jiang, R.; Feliciani, C.; Liu, Y.; Shiwakoti, N.; Li, D. Incentive-based experiments to characterize pedestrians' evacuation behaviors under limited visibility. Saf. Sci. 2021, 133, 105013. [CrossRef]

12. Bernardini, G.; Lovreglio, R.; Quagliarini, E. Proposing behavior-oriented strategies for earthquake emergency evacuation: A behavioral data analysis from New Zealand, Italy and Japan. Saf. Sci. 2019, 116, 295-309. [CrossRef]

13. Chen, X.; Zhan, F.B. Agent-based modelling and simulation of urban evacuation: Relative effectiveness of simultaneous and staged evacuation strategies. J. Oper. Res. Soc. 2008, 59, 25-33. [CrossRef]

14. Li, X.; Li, Q.; Claramunt, C. A time-extended network model for staged evacuation planning. Saf. Sci. 2018, 108, 225-236. [CrossRef]

15. Carvalho, I.; Lewis, D.A. Beyond community: Reactions to crime and disorder among inner-city residents. Criminology 2003, 41, 779-812. [CrossRef]

16. Wilson, J.; Kelling, G. Broken Windows: The police and neighborhood safety. Atl. Mon. 1982, 249, $29-38$.

17. Skogan, W.G. Disorder and Decline: Crime and the Spiral of Decay in American Neighborhoods; The Free Press: New York, NY, USA, 1991; Volume 28.

18. Keizer, K.; Lindenberg, S.; Steg, L. The Importance of Demonstratively Restoring Order. PLoS ONE 2013, 8, e65137. [CrossRef]

19. Chaiechi, T. The broken window: Fallacy or fact-A Kaleckian-Post Keynesian approach. Econ. Model. 2014, 39, 195-203. [CrossRef]

20. Liu, J.; Wu, J.S.; Che, T. Understanding perceived environment quality in affecting tourists' environmentally responsible behaviours: A broken windows theory perspective. Tour. Manag. Perspect. 2019, 31, 236-244. [CrossRef]

21. Nishinari, K.; Kirchner, A.; Namazi, A.; Schadschneider, A. Extended Floor Field CA Model for Evacuation Dynamics. IEICE Trans. Inf. Syst. 2004, E87-D, 726-732.

22. Zheng, Y.; Jia, B.; Li, X.G.; Zhu, N. Evacuation dynamics with fire spreading based on cellular automaton. Phys. A Stat. Mech. Its Appl. 2011, 390, 3147-3156. [CrossRef]

23. Liu, Q. A social force model for the crowd evacuation in a terrorist attack. Phys. A Stat. Mech. Its Appl. 2018, 502, 315-330. [CrossRef]

24. Liu, Q. A social force approach for the defensive strategy of security guards in a terrorist attack. Int. J. Disaster Risk Reduct. 2020, 46, 101605. [CrossRef]

25. Zheng, Y.; Li, X.G.; Jia, B.; Jiang, R. Simulation of pedestrians' evacuation dynamics with underground flood spreading based on cellular automaton. Simul. Model. Pract. Theory 2019, 94, 149-161. [CrossRef]

26. Hoogendoorn, S.P.; Bovy, P.H.L. Pedestrian route-choice and activity scheduling theory and models. Transp. Res. Part B Methodol. 2004, 38, 169-190. [CrossRef]

27. Hoogendoorn, S.P.; Van Wageningen-Kessels, F.; Daamen, W.; Duives, D.C.; Sarvi, M. Continuum Theory for Pedestrian Traffic Flow: Local Route Choice Modelling and its Implications. Transp. Res. Procedia 2015, 7, 381-397. [CrossRef]

28. Helbing, D.; Molnár, P. Social force model for pedestrian dynamics. Phys. Rev. E 1995, 51, 4282-4286. [CrossRef] [PubMed]

29. Helbing, D.; Farkas, I.; Vicsek, T. Simulating dynamical features of escape panic. Nature 2000, 407, 487-490. [CrossRef]

30. Burstedde, C.; Klauck, K.; Schadschneider, A.; Zittartz, J. Simulation of pedestrian dynamics using a two-dimensional cellular automaton. Phys. A Stat. Mech. Its Appl. 2001, 295, 507-525. [CrossRef]

31. Kirchner, A.; Schadschneider, A. Simulation of evacuation processes using a bionics-inspired cellular automaton model for pedestrian dynamics. Phys. A Stat. Mech. Its Appl. 2002, 312, 260-276. [CrossRef]

32. Guo, R.Y.; Huang, H.J. A mobile lattice gas model for simulating pedestrian evacuation. Phys. A Stat. Mech. Its Appl. 2008, 387, 580-586. [CrossRef]

33. Guo, X.; Chen, J.; Zheng, Y.; Wei, J. A heterogeneous lattice gas model for simulating pedestrian evacuation. Phys. A Stat. Mech. Its Appl. 2012, 391, 582-592. [CrossRef]

34. D'Orazio, M.; Spalazzi, L.; Quagliarini, E.; Bernardini, G. Agent-based model for earthquake pedestrians' evacuation in urban outdoor scenarios: Behavioural patterns definition and evacuation paths choice. Saf. Sci. 2014, 62, 450-465. [CrossRef]

35. Li, M.; Zhao, Y.; He, L.; Chen, W.; Xu, X. The parameter calibration and optimization of social force model for the real-life 2013 Ya'an earthquake evacuation in China. Saf. Sci. 2015, 79, 243-253. [CrossRef]

36. Zhang, H.; Liu, H.; Qin, X.; Liu, B. Modified two-layer social force model for emergency earthquake evacuation. Phys. A Stat. Mech. Its Appl. 2018, 492, 1107-1119. [CrossRef] 
37. Zimbardo, P.G. The human choice: Individuation, reason, and order versus deindividuation, impulse, and chaos. In Nebraska Symposium on Motivation; University of Nebraska Press: Lincoln, NE, USA, 1969; pp. 237-307.

38. Baxter, P.J. Individual and Community Responses to Trauma and Disaster: The Structure of Human Chaos. BMJ 1995, 310, 269. [CrossRef]

39. O'Brien, D.T.; Farrell, C.; Welsh, B.C. Broken (windows) theory: A meta-analysis of the evidence for the pathways from neighborhood disorder to resident health outcomes and behaviors. Soc. Sci. Med. 2019, 228, 272-292. [CrossRef] [PubMed]

40. Lovreglio, R.; Fonzone, A.; dell'Olio, L.; Borri, D.; Ibeas, A. The Role of Herding Behaviour in Exit Choice During Evacuation. Procedia Soc. Behav. Sci. 2014, 160, 390-399. [CrossRef]

41. Lin, J.; Zhu, R.; Li, N.; Becerik-Gerber, B. Do people follow the crowd in building emergency evacuation? A cross-cultural immersive virtual reality-based study. Adv. Eng. Inform. 2020, 43, 101040. [CrossRef]

42. Haghani, M.; Sarvi, M. Imitative (herd) behaviour in direction decision-making hinders efficiency of crowd evacuation processes. Saf. Sci. 2019, 114, 49-60. [CrossRef]

43. Prati, G.; Saccinto, E.; Pietrantoni, L.; Pérez-Testor, C. The 2012 Northern Italy Earthquakes: Modelling human behaviour. Nat. Hazards 2013, 69, 99-113. [CrossRef]

44. Couch, S.R. Handbook of Disaster Research; Springer: New York, NY, USA, 2008; Volume 37.

45. Li, Y.; Chen, M.; Dou, Z.; Zheng, X.; Cheng, Y.; Mebarki, A. A review of cellular automata models for crowd evacuation. Phys. A Stat. Mech. Its Appl. 2019, 526, 120752. [CrossRef]

46. Song, W.; Xu, X.; Wang, B.H.; Ni, S. Simulation of evacuation processes using a multi-grid model for pedestrian dynamics. Phys. A Stat. Mech. Its Appl. 2006, 363, 492-500. [CrossRef]

47. Helbing, D.; Johansson, A.; Al-Abideen, H.Z. Dynamics of crowd disasters: An empirical study. Phys. Rev. E Stat. Nonlinear Soft Matter Phys. 2007, 75. [CrossRef] [PubMed]

48. Helbing, D.; Isobe, M.; Nagatani, T.; Takimoto, K. Lattice gas simulation of experimentally studied evacuation dynamics. Phys. Rev. E Stat. Nonlinear Soft Matter Phys. 2003, 67, 4. [CrossRef] [PubMed]

49. Koretz, J.F.; Handelman, G.H. How the human eye focuses. Sci. Am. 1988, 259, 92-99. [CrossRef]

50. Ao, Y.; Huang, K.; Wang, Y.; Wang, Q.; Martek, I. Influence of built environment and risk perception on seismic evacuation behavior: Evidence from rural areas affected by Wenchuan earthquake. Int. J. Disaster Risk Reduct. 2020, 46. [CrossRef]

51. Li, S.; Yu, X.; Zhang, Y.; Zhai, C. A numerical simulation strategy on occupant evacuation behaviors and casualty prediction in a building during earthquakes. Phys. A Stat. Mech. Its Appl. 2018, 490, 1238-1250. [CrossRef]

52. Aalami, S.; Kattan, L. Fairness and efficiency in pedestrian emergency evacuation: Modeling and simulation. Saf. Sci. 2020, 121, 373-384. [CrossRef]

53. Liu, H.; Liu, B.; Zhang, H.; Li, L.; Qin, X.; Zhang, G. Crowd evacuation simulation approach based on navigation knowledge and two-layer control mechanism. Inf. Sci. 2018, 436-437, 247-267. [CrossRef]

54. Wilson, J.Q.; George, L. Kelling: Broken Windows Theory. In Encyclopedia of Criminological Theory; Cullen, F.T., Wilcox, P., Eds.; SAGE Publications, Inc.: Thousand Oaks, CA, USA, 2010. 